\title{
An Adaptive Organization Index to Characterize Atrial Fibrillation using Wrist-Type Photoplethysmographic Signals
}

\author{
Sibylle Fallet ${ }^{1}$, Mathieu Lemay ${ }^{2}$, Philippe Renevey ${ }^{2}$, Célestin Leupi ${ }^{3}$, Etienne Pruvot $^{3}$, Jean-Marc \\ Vesin $^{1}$ \\ ${ }^{1}$ Swiss Federal Institute of Technology, Lausanne, Switzerland \\ ${ }^{2}$ Swiss Center for Electronics and Microtechnology (CSEM), Neuchâtel, Switzerland \\ ${ }^{3}$ Arrhythmia Unit, Heart and Vascular Department, Lausanne University Hospital, Lausanne, \\ Switzerland
}

\begin{abstract}
The performance of photoplethysmography (PPG)based wearable monitors to diagnose atrial fibrillation $(A F)$ remains unknown to date. This study aims at assessing the performance of new indices quantifying the level of organization in PPG signals to diagnose AF. A database made of 18 adult patients undergoing catheter ablation of various cardiac arrhythmias was used. PPG signals were recorded using a wrist-type sensor. A 12-lead ECG was used as gold standard. ECGs were annotated by experts and selected segments were divided into 4 categories: sinus rhythm (SR), regularly paced rhythm (RPR), irregularly paced rhythm (IPR) and AF. The level of organization of the various $P P G$ signals was measured using an adaptive organization index (AOI), defined as the ratio of the power of the fundamental frequency and the first harmonic to the total power of the PPG signal, computed with adaptive band-pass filters. A total of 2806/803/852/287 10-second epochs were considered for AF/SR/RPR/IPR classes. The following mean AOI values were measured: $0.45 \pm 0.11$ for $A F, 0.73 \pm 0.19$ for $S R, 0.78 \pm 0.20$ for $R P R$ and $0.61 \pm 0.19$ for IPR classes. Importantly, the AF AOI was significantly smaller than that of the other categories $(p<0.001)$, indicating a higher degree of disorganization.
\end{abstract}

\section{Introduction}

Atrial fibrillation (AF) is the most common cardiac arrhythmia, affecting $1-2 \%$ of the general population [1]. In Occident, the prevalence of AF varies between $0.1 \%$ among adults younger than 55 year-old to $9.0 \%$ in persons aged 80 years or older [2]. AF can be asymptomatic, hence difficult to diagnose in its early stage. The gold standard for the diagnosis of $\mathrm{AF}$ is the electrocardiogram (ECG). Despite recent progress, portable ECG devices remain costly and too cumbersome for the screening of $\mathrm{AF}$ in large populations. Alternative diagnostic tools are desirable.

Photoplethysmography (PPG) is an optical technique to measure blood volume changes. The pulsatile component of the PPG waveform has proven to be suitable to extract heart rate, among some other cardiovascular parameters [3]. PPG devices have emerged as popular heart rate ambulatory monitors because they can be comfortably worn for long-time periods, during daily activities and are lowcost. However, their ability to diagnose cardiac arrhythmias, such as AF, remains unexplored. Recently, the ability of PPG signals provided by an iPhone $4 \mathrm{~S}$ to detect AF was investigated $[4,5]$. In these studies, the RR intervals were first computed from the PPG waveforms acquired with the camera of the device. Then, various features were derived from the RR-intervals. In [4], an accuracy of 0.968 was achieved to discriminate between irregular pulse (AF) and sinus rhythm, using the Shannon entropy and the root mean square of successive difference of RR intervals on 2minute segments. Using the same parameters on 5-minute segments, the AUC of the ROC measured 0.966 [5].

AF is often described as a chaotic rhythm. On the basis of this observation, studies have been carried out to find measures able to quantify the organization of ECG signals during AF. For instance, the mean-squared error in the linear prediction between two ECGs has been used as a measure of organization [6]. The disorganization of ECG signals occurring during $\mathrm{AF}$ can also be computed from the spectrum of ECG signals [7]. The possibility of deriving such measures from PPG signals to characterize the type of arrhythmia has never been investigated so far.

Our study aims at assessing the performance of new indices quantifying the level of organization in PPG signals to diagnose AF. 


\section{Methods}

\subsection{Data}

The database is made of recordings from 18 patients undergoing catheter ablation of cardiac arrhythmias (Arrhythmia Unit, Heart and Vascular Department, Lausanne University Hospital, Switzerland). The PPG waveforms were acquired with a wrist-type device developed by the Swiss Center for Electronics and Microtechnology (CSEM). This device comprises three infrared LEDs and a 3-axis accelerometer (ACC). The waveforms were sampled at $21.33 \mathrm{~Hz}$. A 12-lead ECG was recorded at $2 \mathrm{kHz}$ (Sensis, Siemens). All ECG segments belonging to one of these four categories were annotated by local experts: sinus rhythm (SR), regularly paced rhythm (RPR), irregularly paced rhythm (IPR) and AF. IPR corresponds to atrial and ventricular pacing protocols with increment in pacing rate. The study was approved by the local ethics committee.

\subsection{Pre-processing of PPG waveforms}

PPG and ECG waveforms were temporally aligned using the inter-beat intervals time series. The ECG RR intervals were derived from the positions of the R-waves, provided by the recording system. The PPG waveform inter-beat intervals were derived from the time-of-arrival of the pulses, computed from the detection of the zeros of curvature (second derivative of the signal). As other external factors non directly related to the heart rate, such as respiration and body motions, also produce changes in the measured optical signals, the direct alignment of these two series was not straightforward. In order to find the best alignment a dynamic time warping (DTW) algorithm was used [8]. More details about the alignment procedure are provided in the complementary study [9].

PPG waveforms were filtered by a finite impulse response filter, with the following coefficients:

$$
d d_{n}=\left[\frac{1}{3}, \frac{1}{3}, \frac{1}{3}, \frac{-2}{3}, \frac{-2}{3}, \frac{-2}{3}, \frac{1}{3}, \frac{1}{3}, \frac{1}{3}\right] \circ \text { hanning(9) }
$$

where $\circ$ denotes the element-wise multiplication and hanning $(9)$ is a hanning window of length 9.

PPG measurements are known to be quite sensitive to motion artifacts [3], hence ACC signals were used to detect and discard motion periods. More specifically, a threshold was put on the mean standard deviation of the three ACC waveforms. Figure 1 illustrates the automatic detection of motion artifacts.

\subsection{Adaptive organization index}

The organization index used in this study is defined as the ratio of the power of the fundamental frequency and

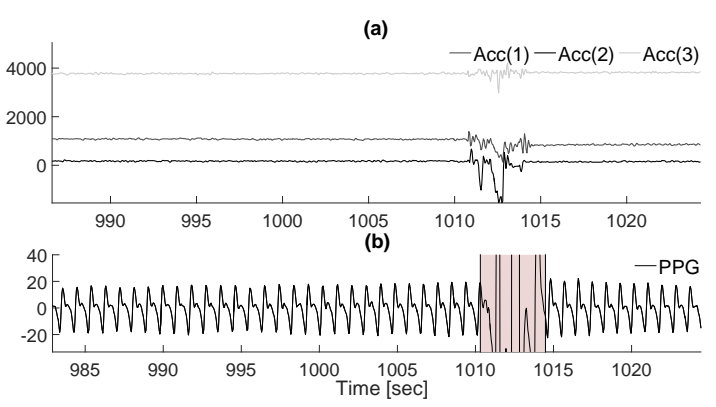

Figure 1. Removal of PPG segments corrupted by motion artifacts. (a) The three-axis ACC signals; (b) PPG waveform and the motion artifact zone detected using the ACC signals.

the first harmonic to the total power of the pre-processed PPG signal. This index, introduced in [7], has been previously used in [10] to track the AF termination using the surface ECGs during stepwise radiofrequency catheter ablation of AF. The authors used adaptive band-pass filters to track the instantaneous fundamental and first harmonic frequency components [10]. The harmonic frequency tracker (HFT), described in [11], is based on time-varying single pole band-pass filters with the following transfer function:

$$
G(z ; n)=\frac{1-\beta}{1-\beta e^{j w[n]} z^{-1}}
$$

with $w[n]$, the normalized instantaneous frequency estimate and $\beta(0 \ll \beta<1)$, a factor related to the bandwidth of the filter. The adaptive mechanism used to update the central frequency of the band-pass filter in (2) at each time step is based on the complex oscillator equation:

$$
c[n]=e^{j w_{0}} c[n-1]
$$

from which the following cost function was derived:

$$
J=E\left\{\left|y[n]-e^{j w[n+1]} y[n-1]\right|^{2}\right\}
$$

The minimization of this cost function leads to the following expression for the update of the central frequency:

$$
w[n+1]=\arg \{E\{y[n] \bar{y}[n-1]\}\}
$$

By considering the following input signal:

$$
x[n]=\sum_{k=1}^{K} c_{k}[n]+v[n]=\sum_{k=1}^{K} A_{k} e^{j k w_{0} n}+v[n]
$$

with $A_{k}$ and $k w_{0}$, the complex amplitudes and frequencies of the cisoids, $\mathrm{K}$ the known number of harmonic components, and $v[n]$, an additive centered complex noise. The adaptive band-pass filter described in (2) is used to extract 
the $k^{t h}$ harmonic component (the central frequency is $k$ times the current estimate of the fundamental frequency $w[n])$. The output signal for the $k^{t h}$ harmonic component is

$$
y_{k}[n]=\beta e^{j k w[n]} y_{k}[n-1]+(1-\beta) x[n]
$$

with $k=1, \ldots, K$. For each of these harmonic components the instantaneous frequency is computed using (5), which can be approximated in practice by:

$$
\begin{gathered}
Q_{k}[n]=\delta Q_{k}[n-1]+(1-\delta) y_{k}[n] \overline{y_{k}}[n-1] \\
w_{k}[n+1]=\frac{\arg \left\{Q_{k}[n]\right\}}{k}
\end{gathered}
$$

with $\delta$, a forgetting factor. A weighting procedure is finally applied in order to estimate the instantaneous fundamental frequency. These two expressions are first computed:

$$
\begin{array}{r}
J_{k}[n]=\delta J_{k}[n-1]+(1-\delta)\left|y_{k}[n]-e^{j k w[n]} y_{k}[n-1]\right|^{2} \\
S_{k}[n]=\delta S_{k}[n-1]+(1-\delta)\left|y_{k}[n]\right|^{2}
\end{array}
$$

The weights are defined as:

$$
W_{k}[n+1]=\frac{S_{k}[n] / J_{k}[n]}{\sum_{i=1}^{K} S_{i}[n] / J_{i}[n]}
$$

Finally, the frequency update is computed as

$$
w[n+1]=\sum_{k=1}^{K} W_{k}[n+1] w_{k}[n+1]
$$

In this study, we used $K=2$. The adaptive organization index (AOI) was then calculated as the ratio of the power of the fundamental frequency and the first harmonic to the total power of the pre-processed PPG signal. For this study, we set $\beta=\delta=0.95$. The power was computed from the low-pass filtered squared extracted components (fundamental and first harmonic). 10-second epochs (50\% overlap) of PPG waveforms were considered and the mean AOI for each epoch was reported.

\section{Results}

A total of 2806/803/852/287 10-second epochs were considered for AF/SR/RPR/IPR classes, respectively. Mean AOI values were the following: $0.73 \pm 0.19$ for SR, $0.61 \pm 0.19$ for IPR, $0.78 \pm 0.20$ for RPR and $0.45 \pm 0.11$ for AF. The AF AOI was significantly lower than that of the other categories (Wilcoxon sum-rank test). These results are displayed in Figure 2. The AUC of the ROC was 0.864 between AF and SR classes. Figure 3 illustrates the extraction of the fundamental and the first harmonic components for two 20-second segments during AF and SR. The resulting AOI is also shown.

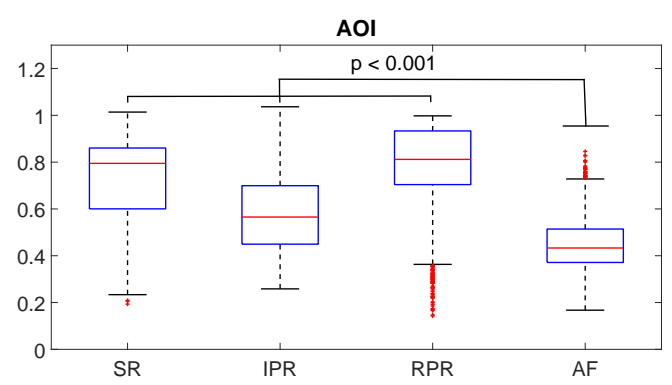

Figure 2. Boxplot of the obtained AOI values for the four classes.

\section{Discussion}

The level of organization of PPG signals during electrophysiology procedures was studied using an index computed directly from the PPG waveform. The mean AOI during regular pacing (RPR) was higher than that during $\mathrm{SR}$, confirming that the investigated AOI was representative of signal organization. Interestingly, we found that the AF AOI was significantly lower than that of the other categories, indicating a higher degree of disorganization during AF.

This AOI was previously investigated in [10] to quantify the amount of oscillations in AF waves from ECG signals, after cancellation of ventricular activity, where the main frequency extracted by the HFT was the frequency of fibrillatory waves. This measure was presented as a measure of AF complexity. However, using PPG signals, this AOI could be seen as a measure of regularity in blood pumping. The AUC of 0.864 to discriminate between SR and AF highlights the potential of AOI as a feature in a classification scheme. Prior studies to characterize AF using PPG were based only on RR intervals-derived parameters $[4,5]$. However, the extraction of RR intervals from PPG signal can be challenging, especially in the presence of several arrhythmias. It is therefore interesting to have a complementary measure based on the waveform itself.

To the best of our knowledge, our results, besides the results of the associated study [9], constitute the first clinical evidence of reliable AF detection using wrist-type PPG device.

For further study, the AOI will be combined to the RRbased measures proposed in [9]. The behavior of the investigated AOI in presence of other types of arrhythmia (ventricular tachycardia, atrial flutter, etc.) should also be examined.

\section{Conclusion}

Our preliminary results suggest that AF is characterized by a higher degree of disorganization of PPG signals sim- 

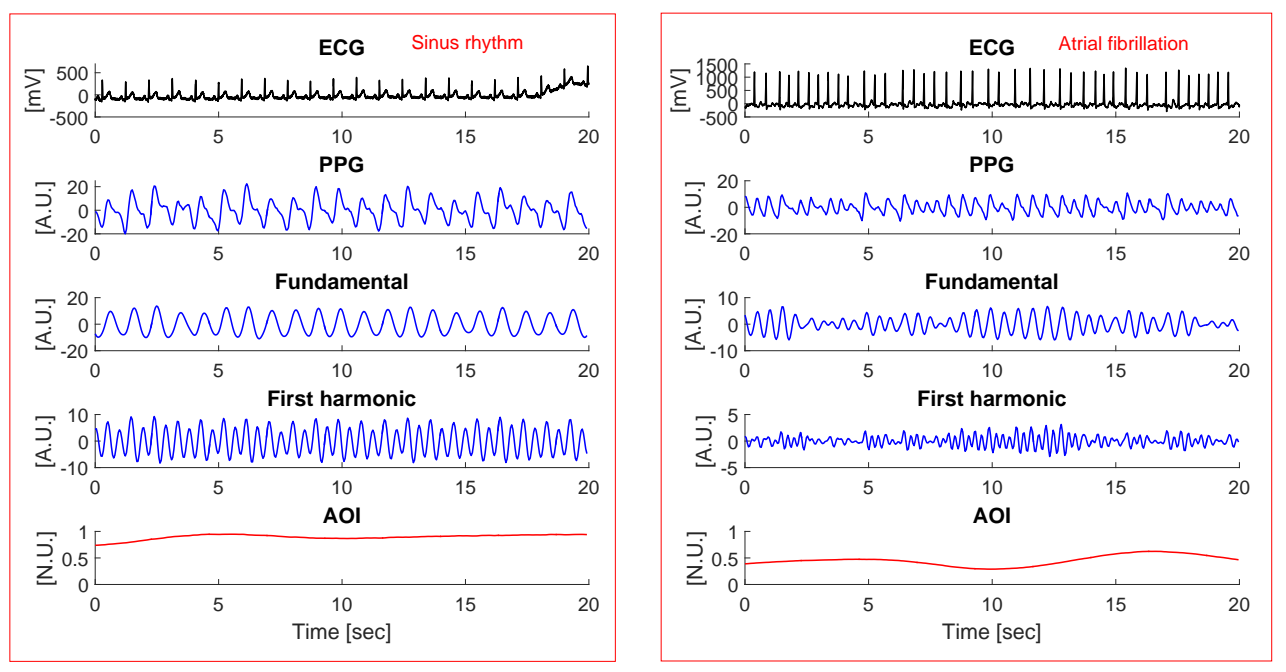

Figure 3. Illustrative example for sinus rhythm and atrial fibrillation segments. (a) ECG waveform; (b) PPG waveform; (c) Fundamental component; (d) First harmonic component; (e) The resulting AOI.

ilarly to that of ECG signals. The AOI is a simple and promising measure of organization. Thanks to their ability to record long periods in a non-invasive and comfortable way, PPG-based wrist monitors appear as promising diagnostic tools for the screening of $\mathrm{AF}$ in large populations.

\section{Acknowledgements}

This work was funded thanks to the Swiss NanoTera initiative, NTF project MiniHolter.

\section{References}

[1] Camm AJ, et al. Guidelines for the management of atrial fibrillation. European Heart Journal 2010;31:2369-2429.

[2] Go AS, Hylek EM, Phillips KA, et al. Prevalence of diagnosed atrial fibrillation in adults: National implications for rhythm management and stroke prevention: the anticoagulation and risk factors in atrial fibrillation (atria) study. JAMA 2001;285:2370-2375.

[3] Allen J. Photoplethysmography and its application in clinical physiological measurement. Physiol Meas 2007;28:R139.

[4] McManus DD, Lee J, Maitas O, Esa N, Pidikiti R, Carlucci A, Harrington J, Mick E, Chon KH. A novel application for the detection of an irregular pulse using an iphone $4 \mathrm{~s}$ in patients with atrial fibrillation. Heart Rhythm 2013;10:315319.

[5] Krivoshei L, Weber S, Burkard T, Maseli A, Brasier N, Khne M, Conen D, Huebner T, Seeck A, Eckstein J. Smart detection of atrial fibrillation. Europace 2016; euw125.
[6] Sih HJ, Zipes DP, Berbari EJ, Olgin JE. A high-temporal resolution algorithm for quantifying organization during atrial fibrillation. IEEE Trans Biomed Eng 1999;46:440450.

[7] Everett TH, Moorman JR, Kok LC, Akar JG, Haines DE. Assessment of global atrial fibrillation organization to optimize timing of atrial defibrillation. Circulation 2001; 103:2857-2861.

[8] Berndt DJ, Clifford J. Using dynamic time warping to find patterns in time series. In KDD workshop, volume 10. Seattle, WA, 1994; 359-370.

[9] Lemay M, Fallet S, Renevey P, Leupi C, Pruvot E, Vesin JM. Wrist-located heart rate monitor device used for atrial fibrillation screening: A preliminary study. In 2016 Computing in Cardiology. September 2016;

[10] Buttu A, Zaen JV, Viso A, Forclaz A, Pascale P, Narayan SM, Vesin JM, Pruvot E. A new method for ECG tracking of persistent atrial fibrillation termination during stepwise ablation. In 2011 Computing in Cardiology. September $2011 ; 165-168$.

[11] Van Zaen J, Uldry L, Duchêne C, Prudat Y, Meuli RA, Murray MM, Vesin JM. Adaptive tracking of EEG oscillations. Journal of Neuroscience Methods 2010;186:97-106.

Address for correspondence:

Sibylle Fallet

EPFL SCI STI JMV - ELD 224 - Station 11

1015 Lausanne, Switzerland

sibylle.fallet@epfl.ch 